\section{¿Se debe revisar la determinación de la CIM de colistín en Acinetobacter baumannii?}

\author{
Xabier Villanueva, Andrés Opazo-Capurro, \\ Mario Quezada-Aguiluz, Mariana Domínguez, \\ Helia Bello-Toledo y Gerardo González-Rocha
}

The colistin MIC determination in Acinetobacter baumannii, Should it be reviewed?

Currently, there is a controversy in how to determine the minimal inhibitory concentration (MIC) of colistin against Acinetobacter baumannii. We compared three methods, concluding that the addition of Tween-80 $(0.002 \%)$ to Müller-Hinton broth in the microdilution method could improve MIC determination and it could reduce false resistance.

Key words: Colistin, Acinetobacter baumannii, MIC determination.

Palabras clave: Colistín; Acinetobacter baumannii; determinación de CIM.

\section{Introducción}

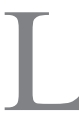

a emergencia de infecciones asociadas a la atención de salud (IAAS) por cepas de Acinetobacter baumannii extensivamente resistentes (XDR, por su sigla en inglés) constituye un gran problema, ya que sólo presentan susceptibilidad in vitro a colistín ${ }^{1}$. Sin embargo, la determinación de la concentración inhibitoria mínima (CIM) mediante microdilución tiene el problema que colistín se adhiere a las paredes de las microplacas de policarbonato, afectando los valores de las CIM $^{2}$. Existe un debate abierto en si se debe corregir este fenómeno adicionando Tween-80 $(0,002 \%)^{3}$, con estudios que apoyan ${ }^{4}$ y otros que rechazan esta indicación ${ }^{5}$, basado en el potencial efecto sinérgico entre el surfactante y colistín. En este trabajo de investigación se evaluó el efecto de la metodología empleada en la determinación de la CIM de colistín sobre cepas del complejo $A$. baumannii (CAB).

\section{Materiales y Método}

Se estudiaron 32 aislados de origen hospitalario del CAB, catalogados previamente como resistentes a colistín mediante microdilución en caldo ${ }^{6}$. Se determinó la CIM de colistín siguiendo recomendaciones del CLSI, con los siguientes métodos: microdilución en caldo en ausencia $[\mathrm{MCS}]^{6} \mathrm{y}$ en

Facultad de Ciencias Biológicas. Universidad de Concepción

Departamento de Microbiología. Laboratorio de Investigación en Agentes Antibacterianos.

Conflictos de interés: Los autores no declaran conflictos de interés.

Financiamiento: Proyectos FONDECYT Nº1130838 y N³150286 y Beca de Magíster № 22140977 a

XVM, de la Comisión Nacional de Investigación Científica y Tecnológica de Chile (CONICYT).

Recibido: 16 de mayo de 2017 / Aceptado: 4 de julio de 2017

Correspondencia a:

Gerardo González-Rocha

ggonzal@udec.cl presencia de Tween-80 (0,002\%) [MCT $]^{3}$; y macrodilución en caldo usando tubos de borosilicato $[\mathrm{MCTB}]^{7}$. El punto de corte para definir resistencia fue una CIM $\geq 4 \mu \mathrm{g} / \mathrm{mL}^{6}$. Como cepa control, se utilizó Pseudomonas aeruginosa ATCC $^{\circledR} 27853$ (rango de CIM de 0,5 a $4 \mu \mathrm{g} / \mathrm{mL}$ ). Adicionalmente, se estudió el efecto del surfactante sobre la viabilidad del aislado A. baumannii A-436 (CIM de $4 \mu \mathrm{g} / \mathrm{mL}$ ) monitoreando su crecimiento (mediante $\left.\mathrm{DO}_{600}\right)$ en caldo Müller-Hinton con Tween-80 $(0,002 \%)$ y en caldo Müller-Hinton con Tween-80 (0,002\%) adicionado de $2 \mu \mathrm{g} / \mathrm{mL}$ de colistín (concentración subinhibitoria).

\section{Resultados}

La CIM varió entre 4-64 $\mu \mathrm{g} / \mathrm{mL}$ para MCS, $1-32 \mu \mathrm{g} / \mathrm{mL}$ para MCT y 0,5-64 $\mu \mathrm{g} / \mathrm{mL}$ para MCTB. Al comparar con el método MCS, se encontró que $12,5 \%$ (4/32) y 18,8\% (6/32) de las cepas varió de categoría resistente a susceptible al usar MCT y MCTB; respectivamente; y la $\mathrm{CIM}_{50}$ y CIM $_{90}$ disminuyeron de 64 a 8 y $16 \mu \mathrm{g} / \mathrm{mL}$ con el método MCT y a 8 y $32 \mu \mathrm{g} / \mathrm{mL}$ con MCTB. Además, en más de $60 \%$ de los aislados la CIM disminuyó en $\geq 2$ diluciones con los métodos MCT y MCTB.

El crecimiento de $A$. baumannii no fue afectado en presencia Tween- 80 y Tween-80 adicionado de una concentración subinhibitoria de colistín, no observándose diferencias con respecto al crecimiento en ausencia de surfactante.

\section{Discusión}

Comparando los métodos, los valores de CIM obtenidos por MCT y MCTB se asemejan más entre sí, que con los obtenidos por MCS. Sumado al hecho que la adición de Tween- 80 no afectó la viabilidad de $A$. baumannii, indicaría que las CIM obtenidas por MCTB y MCT serían más certeras que aquellas obtenidas por microdilución convencional en caldo [MCS $]^{4}$. No obstante, el CLSI no incorpora Tween- 80 como coadyuvante para la determinación de CIM por microdilución, como sí lo hace en el caso del antibacteriano glucopéptido telavancina ${ }^{6}$.

Por otro lado, se han planteado dos principales mecanismos de resistencia a colistín, uno de ellos por sustitución del lipopolisacárido (LPS) por fosfolípidos (mutación de genes $l p x)^{8}$, y otro por adición de fosfoetanolamina al lípido A del LPS, ya sea por mutación del gen $p m r B^{8}$ o por adquisición de un plásmido portador de $m c r-1^{9}$. En este sentido, sería necesario constatar en futuras investigaciones el tipo de mecanismo de resistencia presente en aislados clínicos chilenos.

Podemos concluir que es necesario profundizar el estudio de la correcta determinación de la CIM de colistín sobre cepas del CAB, debido a que la metodología actual podría estar informando aislados falsamente resistentes. Esto lleva a descartar colistín, que in vitro mostraría ser activo, y sería el único antimicrobiano para tratar infecciones por cepas XDR de esta bacteria.

Agradecimientos: A Sergio Vargas y Daniela Gutiérrez del Hospital de Urgencia Asistencia Pública; Francisco Silva, Marcela Cifuentes y Boris Barrera del Hospital Clínico de la Universidad de Chile y Patricia García del Hospital Clínico de la Universidad Católica, quienes proveyeron gentilmente las cepas incluidas en este estudio. 


\section{Referencias bibliográficas}

1.- Aguayo A, Mella S, Riedel G, Bello H, Domínguez M, González-Rocha G. Colistín en la era post-antibiótica. Rev Chilena Infectol 2016; 33: 166-76.

2.- Sader H S, Rhomberg P R, Farrell D J, Jones R N. Differences in potency and categorical agreement between colistin and polymyxin B when testing 15,377 clinical strains collected worldwide. Diagn Microbiol Infect Dis 2015; 83: 379-81.

3. Sader H S, Rhomberg P R, Flamm R K, Jones R N. Use of a surfactant (polysorbate 80 ) to improve MIC susceptibility testing results for polymyxin B and colistin. Diagn Microbiol Infect Dis 2012; 74: 412-4.

4.- Hindler J A, Humphries R M. Colistin MIC variability by method for contemporary clinical isolates of multidrug-resistant Gram-negative bacilli. J Clin Microbiol 2013; 51: 1678-84.

5.- Brown M R, Winsley B E. Synergism between polymyxin and polysorbate 80 against Pseudomonas aeruginosa. J Gen Microbiol 1971; 68: 367-73.
6.- Clinical and Laboratory Standards Institute (CLSI). Performance Standards for Antimicrobial Susceptibility Testing. 27th ed. CLSI supplement M100. Wayne, PA: Clinical and Laboratory Standards Institute; 2017.

7.- Clinical and Laboratory Standards Institute (CLSI). Methods for Dilution Antimicrobial Susceptibility Tests for Bacteria That Grow Aerobically; Approved Standard-Ninth Edition. CLSI document M07-A9. Wayne, PA: Clinical and Laboratory Standards Institute; 2012.

8.- Beceiro A, Moreno A, Fernández N, Vallejo J A, Aranda J, Adler B, et al Biological cost of different mechanisms of colistin resistance and their impact on virulence in Acinetobacter baumannii. Antimicrob Agents Chemother 2014; 58: 518-26.

9.- $\quad$ Liu Y Y, Wang Y, Walsh T R, Yi L X, Zhang R, Spencer J, et al. Emergence of plasmid-mediated colistin resistance mechanism MCR-1 in animals and human beings in China: a microbiological and molecular biological study. Lancet Infect Dis 2016; 16: 161-8. 